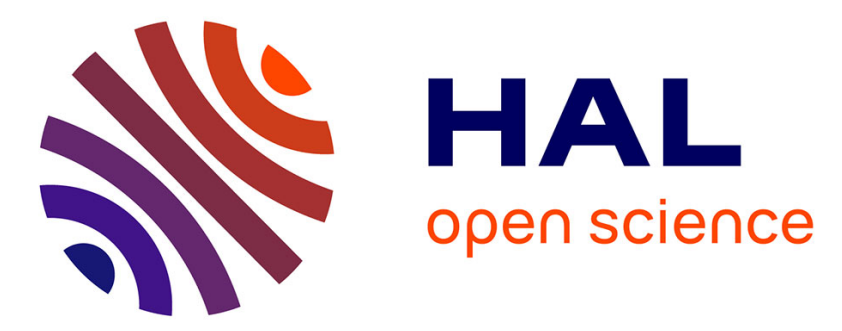

\title{
A signal processing approach for enhanced Acoustic Emission data analysis in high activity systems: application to Organic Matrix Composites
}

Mohamed Kharrat, Emmanuel Ramasso, Vincent Placet, Lamine Boubakar

\section{- To cite this version:}

Mohamed Kharrat, Emmanuel Ramasso, Vincent Placet, Lamine Boubakar. A signal processing approach for enhanced Acoustic Emission data analysis in high activity systems: application to Organic Matrix Composites. Mechanical Systems and Signal Processing, 2016, 70-71, pp.1038 - 1055. hal01303394

\section{HAL Id: hal-01303394 \\ https://hal.science/hal-01303394}

Submitted on 18 Apr 2016

HAL is a multi-disciplinary open access archive for the deposit and dissemination of scientific research documents, whether they are published or not. The documents may come from teaching and research institutions in France or abroad, or from public or private research centers.
L'archive ouverte pluridisciplinaire HAL, est destinée au dépôt et à la diffusion de documents scientifiques de niveau recherche, publiés ou non, émanant des établissements d'enseignement et de recherche français ou étrangers, des laboratoires publics ou privés. 


\title{
A signal processing approach for enhanced Acoustic Emission data analysis in high activity systems: application to Organic Matrix Composites
}

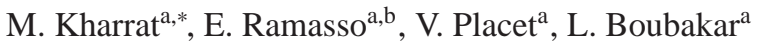 \\ ${ }^{a}$ Department of Applied Mechanics \\ ${ }^{b}$ Department of Automatic Control and Micro-Mechatronic Systems \\ FEMTO-ST Institute - UMR CNRS 6174 - UFC / ENSMM / UTBM, 25000, Besançon, France
}

\begin{abstract}
In Acoustic Emission (AE) applications, the processing of continuous signals resulting from high AE rates or the superimposition of transients emitted from different emission sources, is a major problem. In complex systems like Organic Matrix Composites (OMC) fatigue tests, a high AE activity is produced due to the emergence of several emission sources. Such a kind of tests often involves a lot of noise that corrupts the original signal. Conventional threshold-based techniques are highly influenced by the noise level leading to erroneous hits detection. A suitable denoising method is thus necessary to process the signal before performing the hits detection and separation.

This work deals with the processing of continuous AE signals obtained from Carbon Fiber Reinforced Plastics (CFRP) specimens under complex loading. When the size of each acquisition is large due to the sampling rate (generally from 2 to $5 \mathrm{MS} / \mathrm{s}$ ), the signal is divided into short segments. The Discrete Wavelet Transform (DWT) is then used for signal denoising. An adjustment of its input parameters is achieved in order to improve the denoising process. A hit determination is thereafter performed in order to localize potential hits contained in each signal segment. By comparing the result obtained using the proposed approach to that of the usual threshold-based technique, we remark that the problem of erroneous hits is overcome. The performance of the proposed approach as well as the sensitivity to the denoising parameters are evaluated by studying the impact of errors in hit detection on feature extraction and damage assessment based on pattern recognition algorithms. The proposed approach ensures a better identification of natural clusters in AEs and improves the interpretation of damage mechanisms.
\end{abstract}

Keywords: Continuous AE signals, CFRP, Quasi-static tests, Wavelet denoising, Hit detection, Pattern recognition.

\section{Introduction}

Carbon Fiber Reinforced Plastics (CFRP) materials are increasingly used in aeronautical and automotive industries as well as in civil engineering thanks to their high material properties [1-3]. Since the integrity of CFRP structures needs to be regularly assessed, the Acoustic Emission (AE) technique is widely used to detect and localize eventual damages [4-7]. AE is a non-destructive method able to ensure in-situ monitoring of the structure through a network of distributed sensors, and can be used to detect damages at a very early stage well before the structure becomes completely failed [8]. When the structure is subjected to mechanical, thermal or chemical solicitations, a stress field is generated in the material. As a consequence of the repetition of these solicitations, the material degrades. The appearance of defects leads to the creation of elastic ultrasonic waves that propagate through the material. This wave propagation involves surface vibrations, which are measurable using appropriate sensors. AE consists hence in a transient elastic energy release in materials when microstructural changes occur. It is dependent on some basic deformation and damage mechanisms. In CFRP composites, major damage mechanisms are delamination, matrix cracking, debonding, fiber cracking, and fiber pull-out [9].

\footnotetext{
*Corresponding author. Tel.:+33 381666015 .

Email address: mohamed.kharrat@femto-st.fr (M. Kharrat)
} 
Three types of AE transients can be distinguished: bursts, continuous and mixed [10, 11]. Bursts are generated by defects according to the damage mechanisms and have shorter durations than the other types of transients. Continuous AE transients are created when multiple signals emitted from different emission sources overlap in such a way that the amplitude do not fall below the threshold level. The original transients are often hardly recovered and assigned to their emission sources. The background noise and rubbing in the structure are the main sources of continuous emission. Relevant AE information might be buried by noise preventing the identification of particular damages. Mixed AE transients combine bursts and continuous signals. They can be provoked by damage growth and accumulation and are often superimposed with ambient noise and rubbing [12,13]. This latter type of AE transients is frequently encountered in CFRP structures under complex loading, where the material can be subjected to various solicitations simultaneously (multi-axial stresses, frictions between the clamping jaws and the specimen...) [14]. A high AE activity can be encountered hence, especially when the material is solicited at a high loading rate as well as when its geometry is complex. On one hand, a high loading rate is produced particularly when the cycling frequency is high. Some experimental tests have demonstrated that the AE-hit-rate increases not only with the total number of load cycles but also with the cycling frequency [15]. For highest rates, transients emitted from different emission sources can be superimposed. On the other hand, the inhomogeneity of such a material (fibers, matrices, multiple plies...) engenders anisotropy in the wave velocities. The wave reflections and attenuation (dispersion, geometric spreading...) are also added to the complexity. Complications include steering of the direction of the group velocity caused by the anisotropy of the material, wave attenuation due to damping by the matrix and wave scattering engendered by the fibers [16].

Under in-service conditions, the background noise is sometimes so important that it cannot be neglected. Several sources of perturbation can be the cause. In this study, the noise generated by the hydraulic system of mechanical testing machines is assimilated to a source of perturbation in real operating conditions. The hydraulic fluid is increasingly hot in such machines when the duration of test is long. Irregular flow characteristics and pressure waves propagate through the fluid. A lot of fluidborne noise can be so generated leading to force fluctuations. These result in a vibration, known as structureborne noise, transmitted through the structure [17]. The AE signals can be affected by this noise when it hides them partially or, in many cases, completely. When the level of noise is permanently exceeding the $\mathrm{AE}$ detection threshold, which is the case in continuous emission, the AE system is obliged to terminate the hit after a predefined maximum duration. This latter is configured in the AE system in order to avoid recording long signals. As the threshold is permanently exceeded in such a situation, the AE signal is entirely recorded without a correct hit separation. The consequent AE features can be so affected and the footprint of the noise is not negligible. Most of the commercial parameter-based AE systems employ the conventional threshold-based technique for hits detection and determination. The AE features are calculated without an efficient consideration of noise variations that can mislead the interpretation of real AE events happening in the material. The AE systems also employ predefined band-pass filters in order to avoid the impact of external perturbations. However, if the noise is generated at frequencies that are comprised within the frequency band of the filter, it cannot be suppressed. Thus, the conventional threshold-based technique could not be suitable when dealing with continuous emission, without a further signal processing.

The approach proposed in this study is based on denoising the recorded AE signals prior to the hits determination. The Discrete Wavelet Transform (DWT) is one of the powerful methods that has been widely employed for signal denoising, which has been useful for improving the signal-to-noise ratio much better than using signal filtering, as well as for signal processing to detect multiple defect signatures [18-20]. The DWT is based on the Wavelet Transform (WT) theory [21, 22], which provides relevant information about non-stationary signals in the time-frequency domain. The WT has been used in many studies related to the Structural Health Monitoring (SHM) field [23-28]. Some other studies have reported on the use of the WT on AE signals for a denoising purpose [29-32].

This work proposes a signal processing approach for the purpose of conditioning AE signals issued from continuous emission caused by ambient noise and high AE-hit-rate. The efficiency of the proposed approach is evaluated on experimental AE signals obtained from PLBs performed on CFRP specimens in a noisy environment with different levels, and from a quasi-static test at a high speed of solicitation under a high noise level. The improvement in the AE data analysis by the proposed approach is emphasized.

\section{Description of the AE signal processing approach}

Figure 1 describes the proposed approach as implemented using a Matlab code. The entire AE signal is postprocessed through several steps. It is either handled one shot if it is small in size, or partitioned into equal time- 
segments when its size is large. Massive data signals can be encountered at a high sampling rate with a long acquisition duration. Signals are then denoised using the DWT with appropriate denoising parameters to enhance the signals quality. The conventional process of hits determination and AE features extraction is thereafter performed. The final step is the data clustering, which involves classifying the AE hits into groups (clusters) representing the AE sources.

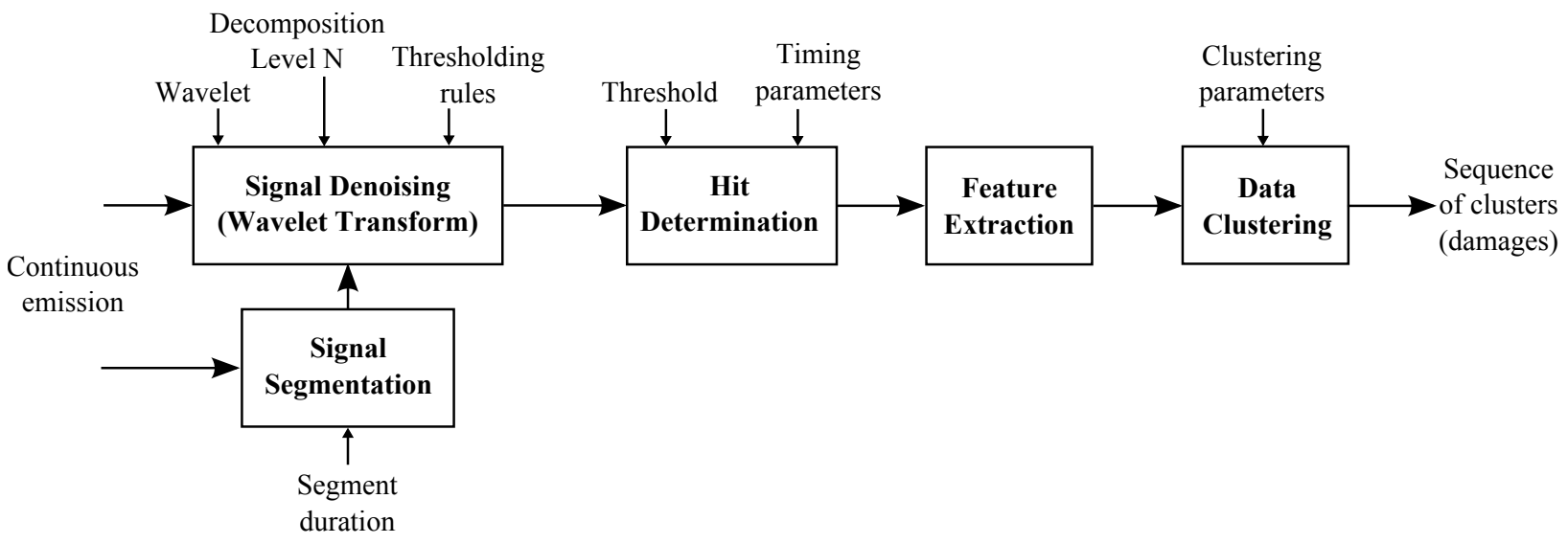

Figure 1: Principle of the proposed AE signal processing approach.

\subsection{Wavelet signal denoising}

Denoising using wavelets is based on the WT theory, which uses a family of wavelets to obtain inner products of the signal to be denoised $s(t)$. A family of wavelets consists of a series of son wavelets generated by dilating and translating a mother wavelet. The wavelet transform $W(a, b)$ gives the so-called wavelet coefficients, which can be considered as functions of translation $b$ (shift or time location) with each fixed scale factor $a$. They give information about the signal $s(t)$ at different levels of resolution and also measure the similarity between $s(t)$ and each son wavelet. In fact, the WT is the convolution between $s(t)$ and the wavelet function, i.e. a wavelet can be used for feature discovery if the chosen wavelet is similar to the feature components hidden in the signal.

The procedure of DWT signal denoising used in this work includes three steps. Firstly, the signal is decomposed up to a defined decomposition level $N$. The detail coefficients are thus obtained. Secondly, a thresholding is performed on the $N$ signal details using a threshold selection rule (fixed form, Stein's Unbiased Risk Estimate principle...) and either a soft or hard thresholding [33,34], by considering a basic model of the noise. Finally, the signal is reconstructed using the original approximation coefficients of the $N^{\text {th }}$ level and the modified detail coefficients of all levels.

The selection of the wavelet function in many engineering applications has been based on trial and error [21]. On one hand, when choosing the wavelet function for SHM applications, it is important to consider its ability to perform the DWT. On the other hand, no unique choice can be recommended for all SHM applications. Some functions might be better used in certain situations than others. The choice of the wavelet function is application dependent and requires careful scrutiny in its use and its results.

\subsection{Hits determination}

In the parameter-based $\mathrm{AE}$ systems [35], the hits detection is performed by comparing the signal to a defined threshold, which is set beforehand above the noise level. The threshold is either fixed during the test (stationary noise), or floating within a defined range (under conditions of high and varying background noise) [36, 37]. In the conventional threshold-based method, whenever the signal surpasses the threshold, the hit detection and determination are carried out by considering three timing parameters: Peak Definition Time (PDT), Hit Definition Time (HDT) and Hit Lockout Time (HLT). The hit determination involves, defining the time-start, the true peak and the time-end of the detected hit. The AE features can be then extracted from a given AE signal $s(t)[8,38]$. The conventional features include Amplitude, Duration, Counts, Counts-to-peak, Rise time, RMS, ASL, PAC-Energy, Signal Strength and Absolute Energy. Frequency features are Average Frequency, Frequency Centroid, Peak Frequency, Initiation 
Frequency, Reverberation Frequency and Partial Power features. Hereinafter, the definitions of some AE features whose the expressions have not been explicitly provided in the literature:

- PAC-Energy $\left(E_{P A C}\right)[\mu \mathrm{V} . \mathrm{s}]:$ A scaled version of Signal Strength to match the analog AE systems with $20 \mathrm{~dB}$ of gain. It is also called MARSE energy (Measured Area under the Rectified Signal Envelope). The sum of the signal envelope is calculated over the duration of the AE hit and converted into counts at $100 \mathrm{kHz} / \mathrm{V}$. PAC-Energy is expressed as:

$$
E_{P A C}=\sum_{t}|H[s(t)]| \cdot \Delta t \cdot 100 \cdot 10^{6},
$$

where $H$ is the envelope - calculated by the Hilbert Transform - of the signal above the threshold.

- Signal Strength $(S t r)[p V . s]$ : It is the integral of the rectified voltage signal over the duration of the AE hit and can be defined as:

$$
S t r=\sum_{t=1}^{L}|s(t)| \cdot \Delta t \cdot 10^{12} .
$$

- Absolute Energy $\left(E_{a b s}\right)[\mathrm{aJ}]$ : The integral of the squared voltage signal above the threshold divided by the reference resistance $(10 \mathrm{k} \Omega)$ over the duration of the $\mathrm{AE}$ hit.

$$
E_{a b s}=\sum_{t=1}^{L}|s(t)|^{2} \cdot \Delta t / R \cdot 10^{18} .
$$

\subsection{Data clustering}

The clustering algorithm used in this study has been previously proposed by the authors [39]. It is based on the idea that several parameterizations may be suitable for a given dataset but it is difficult to precisely find an optimal one. Therefore, the user can provide to it multiple parameterizations: type of algorithm (e.g. Kmeans, FCM, Gustfason-Kessel, Hidden Markov Models), parameters of those algorithms (e.g. type of distance, fuzziness parameter), all possible (and relevant) combinations of features, and so on. Given those parameterizations, a criterion is optimized based on the entropy of the cumulated appearance of clusters which allows getting a subset of relevant parameterizations. The partitions (sequence of clusters) obtained with those parameterizations are then combined. The number of clusters is finally optimized based on an information-theoretic criterion called Normalized Mutual Information NMI [40]. This clustering fusion algorithm is also able to quantify the uncertainty around the estimation of the clusters. This algorithm has been studied with different clustering methods (Kmeans, GK and Hidden Markov Models) on simulated and real datasets. It has been shown that the GK algorithm [41] provided more relevant results. It accounts for its use in the present paper.

\section{Experimental procedure}

\subsection{Materials and methods}

A unidirectional CFRP ring with an outer diameter of $124 \mathrm{~mm}$, a thickness of $1.5 \mathrm{~mm}$ and a width of $16 \mathrm{~mm}$ is used in the experiments (see Figure 2). The mounting is performed according to the ASTM D2290 standard test procedure for apparent hoop tensile strength of plastic or reinforced plastic pipe by split disk method. The ring is fixed using two clamping jaws consisting of two separate half-cylinders. Four Micro80-type (Mistras Group Ltd.) sensors are mounted directly on the clamping jaws using spring clamps to ensure a permanent contact. Sensors 1 and 4 are on the upper half-cylinder, whereas sensors 2 and 3 are on the lower one. It was decided to deport the sensors away from the composite ring in order to avoid their deterioration during the test. Indeed, unidirectional CFRP have highly energetic failure that can damage the sensors if they are placed on the specimen. Thus, acoustic waves pass through the interface between the specimen and the jaws before being captured by the sensors. Potential wave reflections should be so expected. The AE sensors are wide-band and have an operating frequency-range of [200-900 kHz] and a resonant frequency of $325 \mathrm{kHz}$. The AE system settings are shown in Table 1. 


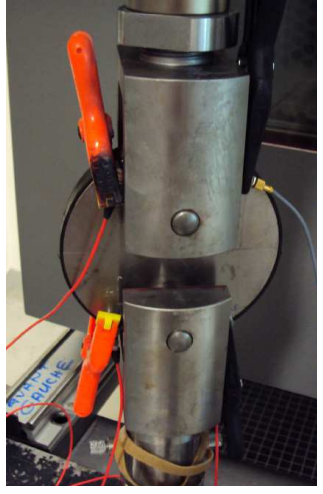

(a)

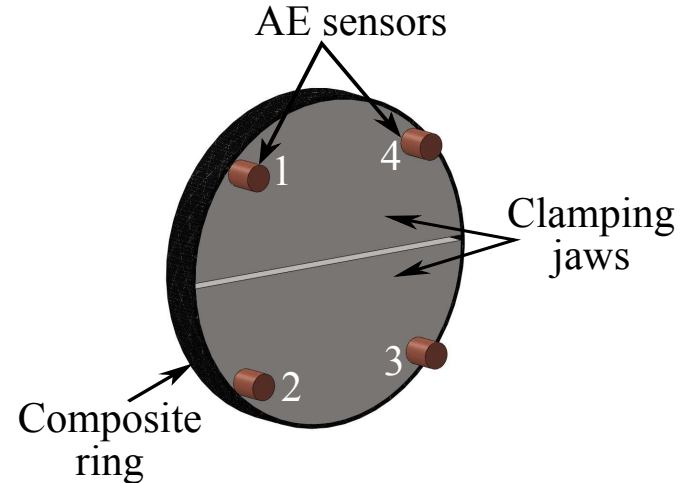

(b)

Figure 2: Configuration of the tests performed on a CFRP ring: (a) a photograph of the mounting on the testing machine; (b) a basic diagram of the mounting.

Table 1: Settings of the AE system.

\begin{tabular}{lc}
\hline Threshold & $40 \mathrm{~dB}$ \\
Pre-Amplifier & $20 \mathrm{~dB}$ \\
Analog Filter & $20 \mathrm{kHz}-1 \mathrm{MHz}$ \\
Sampling Rate & $2 \mathrm{MS} / \mathrm{s}$ \\
PDT & $60 \mu \mathrm{s}$ \\
HDT & $120 \mu \mathrm{s}$ \\
HLT & $300 \mu \mathrm{s}$ \\
Max. Duration & $200 \mathrm{~ms}$ \\
\hline
\end{tabular}

The aim is to recover AE transients from simple and complex signals. To this end, simple AE bursts are firstly generated using Pencil Lead Breaks (PLBs) on the outward surface of an intact specimen under low and high noise levels created by the hydraulic system of the testing machine. As the hydraulic system is located at the bottom of the machine, sensors 2 and 3 are intended to be more affected by the generated background noise. A PLB is the $\mathrm{Hsu}$-Nielsen source frequently employed to simulate an AE event. The fracture of a brittle graphite lead generates an intense acoustic signal, quite similar to a natural AE source, that the sensors detect as a strong burst [42]. These simple AE signals are created in the material while the machine is at rest (no applied loading). A comparison is then addressed between raw and denoised signals in order to demonstrate the efficiency of the approach. The footprint of the noise on the AE features is studied. Thereafter, a tensile test at a high loading rate under a high noise level is performed on the same specimen until its total failure. The tensile force is applied using a hydraulic jack linked to the lower clamping jaw. This experimental configuration is then complex as it involves a multi-axial stress state at the level of the gap between the half-cylinders, frictions between the specimen and the clamping jaws, as well as the consideration of the deported sensors. This results in complex AE signals with a high hit-rate similar to that obtained under in-service conditions. By synthesizing the experiments, three types of signals will be considered:

- Simple AE signal 1: a PLB under a low noise level.

- Simple AE signal 2: a PLB under a high noise level.

- Complex AE signal: a high loading-rate tensile test under a high noise level.

The effectiveness of the implemented approach is assessed by comparing some AE features determined by the algorithm to those obtained by a commercial AE system. 


\subsection{Simple AE signal 1: PLB-burst with a low noise level}

As the channels 2 and 3 are mounted closer to the hydraulic system than channels 1 and 4 , they are subjected to a higher perturbation. Under a low noisy environment, the AE signals received by the four channels are not noticeably impacted, as the threshold is higher than the noise level. Figures 3 and 4 show the Duration-Amplitude graphs and time-signals, respectively, of the hits detected by channels 2 and 3. A further analysis of the measured signals from all channels and their corresponding Duration-Amplitude graphs can be found in a previous publication of the authors [43].

For the purpose of reducing the impact of noise, the raw signals of channels 2 and 3 are denoised using the DWT before performing the hit detection. The parameterization of the denoising procedure is important to enhance the quality of the denoising process [33] and is application-dependent. When dealing with unknown noise level, it would be convenient to adopt a soft thresholding with a selection rule of the universal threshold (denoted 'sqtwolog') equal to $\sqrt{2 \log (n)}$, where $n$ is the total number of wavelet coefficients, and by considering a non-white noise model [34]. This has been confirmed by testing this parameterization on our experimental signals. Another important issue is the choice of the mother wavelet and its order as well as the decomposition level. The Daubechies wavelet [44, 45] is found to be efficient to reduce the noise level in the recorded signals processed in this work. Various orders of the Daubechies wavelet with different decomposition levels $(D L)$ are thereafter tested on the raw signals. In order to evaluate the performance of the signal denoising, the number of detected hits $\left(N_{\text {hits }}\right)$ and the signal-to-noise ratio ( $S N R$ ) between the obtained denoised signal and the residual noise removed from the raw signal are calculated for each tested parameterization. Table 2 summarizes the obtained values for the signals of channels 2 and 3.

Table 2: Denoising performance evaluation of the signals of channels 2 and 3 affected by a low noise level, with " $d b x$ " standing for the $x$-order of the Daubechies wavelet and " $D L$ " for the decomposition level.

\begin{tabular}{clllll} 
& & \multicolumn{2}{l}{ Channel 2 } & \multicolumn{2}{l}{ Channel 3 } \\
\cline { 2 - 6 } & & $N_{\text {hits }}$ & $S N R[d B]$ & $N_{\text {hits }}$ & $S N R[d B]$ \\
\hline Raw signals & 1772 & - & 2020 & - \\
\hline \multirow{3}{*}{$d b 4$} & $D L=4$ & 61 & 3.387 & 1 & 1.169 \\
& $D L=6$ & 1 & 2.316 & 2 & 0.706 \\
& $D L=8$ & 1 & 2.296 & 2 & 0.698 \\
\hline \multirow{3}{*}{$d b 6 \quad D L=4$} & 66 & 3.396 & 1 & 1.180 \\
& $D L=6$ & 1 & 2.313 & 1 & 0.706 \\
& $D L=8$ & 1 & 2.299 & 1 & 0.699 \\
\hline \multirow{3}{*}{$d b 8$} & $D L=4$ & 51 & 3.398 & 1 & 1.187 \\
& $D L=6$ & 1 & 2.308 & 1 & 0.707 \\
& $D L=8$ & 1 & 2.297 & 1 & 0.701 \\
\hline
\end{tabular}

In order to choose the best parameterization, $N_{\text {hits }}$ and $S N R$ must be as small as possible. Indeed, by applying a single PLB, generally one hit or at most few hits should be detected. Besides, the higher is the extracted noise from the raw signal, the lesser is the $S N R$. For the sake of simplicity, the same parameterization is adopted for denoising the signals of both channels 2 and 3. The best parameterization enhancing the quality of these signals is obtained with the " $d b 8$ " wavelet and 8 decomposition levels. As we can see in Figure 4, the noise level is reduced under the threshold for the channels 2 and 3 . Consequently, the number of the detected hits is decreased to only one hit for each channel (Table 2).

\subsection{Simple AE signal 2: PLB-burst with a high noise level}

When the hydraulic fluid reaches an important fluctuations' activity, a high noise is generated in the testing machine. Another PLB is created near sensor 1. As we can see in Figure 5, all the hits detected by the AE system are saturated at a duration of $200 \mathrm{~ms}$, which corresponds to the predefined maximum duration. Thus, the hits separation and the calculated $\mathrm{AE}$ features performed by the $\mathrm{AE}$ system are erroneous. The raw time-signals corresponding to the four channels (see Figure 6) show that the recorded bursts are so noisy that the amplitude never drops below the threshold during the acquisition period, especially for channels 2 and 3. 


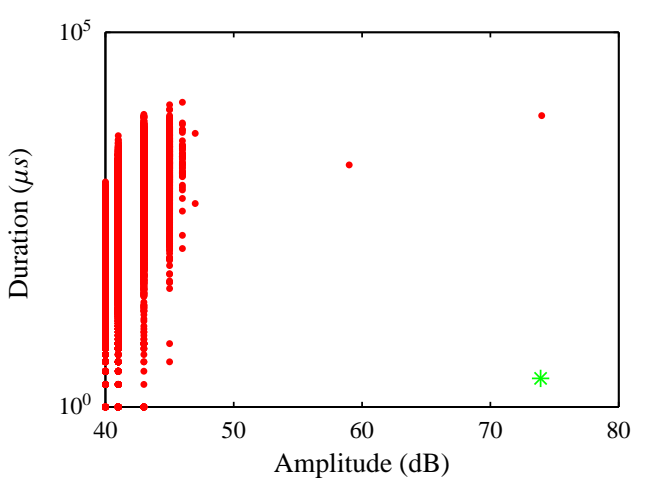

(a) Channel 2 .

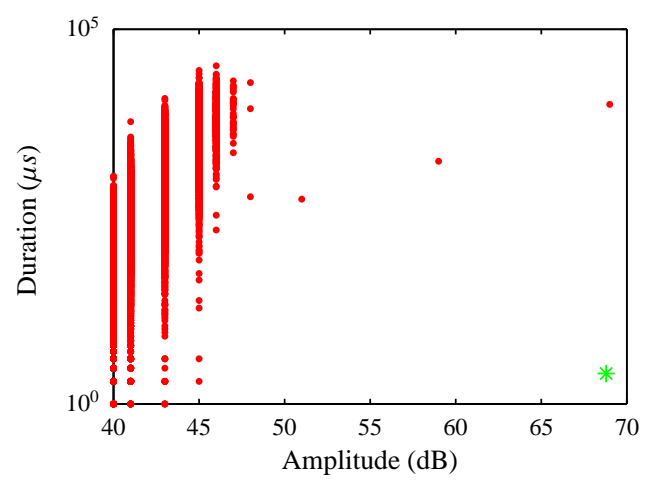

(b) Channel 3 .

Figure 3: PLB bursts under a low noise level: Duration vs. Amplitude graphs of the detected hits by the AE system (•) and by the proposed approach $(*)$. This figure shows that the number of AE hits on channel 2 and 3 is much higher than expected due to the noise level.

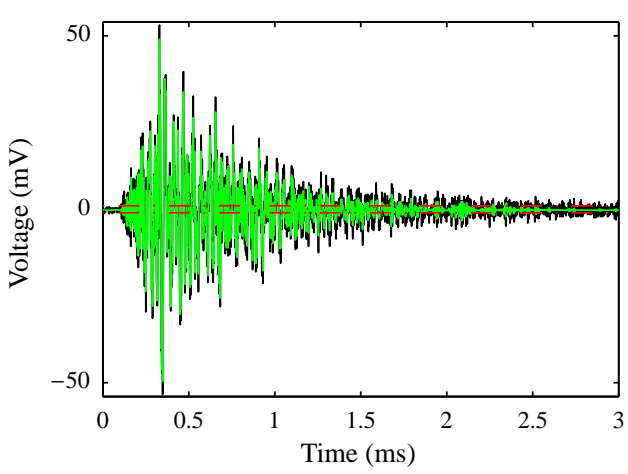

(a) Channel 2

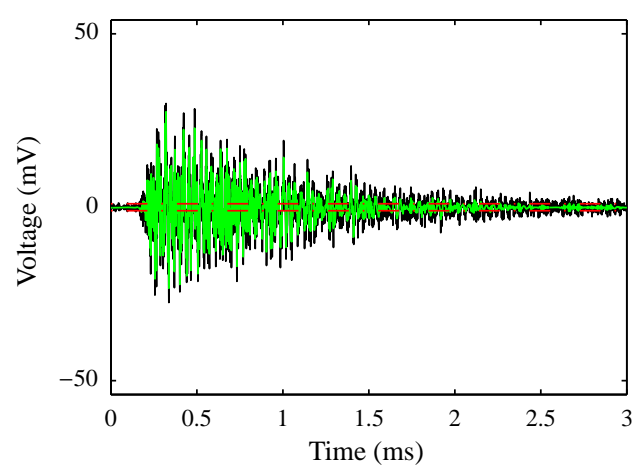

(b) Channel 3 .

Figure 4: [Color representation] Comparison of the raw and denoised signals of channels 2 and 3 after a PLB under a low noise level. Denoising using the " $d b 8$ " wavelet and 8 decomposition levels. (_- Raw signals; (_ $(-)$ denoised signals; and (_- - ) threshold level.

Each channel's signal is denoised using various orders of the Daubechies wavelet with different decomposition levels in order to find the best parameterization. The performance evaluation of the denoising is presented in Table 3 as performed in the last subsection. The results for channels 2 and 3 are presented here as they are more affected by the noise than channels 1 and 4.

Consequently, a good quality of denoising can be obtained using the " $d b 45$ " wavelet with 14 decomposition levels for both channels. This parameterization is also applied on the signals of channels 1 and 4. Figure 6 shows a comparison of the raw and denoised signals of the four channels. As we can see, a considerable improvement of the signals' quality is made, especially for channels 2 and 3. The waveform of the PLB burst is well recovered from the noise. The resulting Duration-Amplitude graphs are represented in Figure 5 where it can be remarked that the saturation of the hits at the predefined maximum duration is now eliminated and the PLB bursts can be identified.

Another issue can be also addressed here, which is the footprint of the noise on the AE features. For that purpose, the frequency contents of the detected hits in both raw and denoised cases are examined. Figure 7 shows the timefrequency diagrams - obtained by the Wavelet Transform (WT) - of the raw and denoised signals of channel 2. As we can see, the frequency content of the hit associated to the PLB-burst in the raw signal is very affected by the noise. We can point out that the denoising process has considerably eliminated the noise contribution in the frequency content of the signal, whereas that of the PLB-burst is kept. Figure 7(a) allows to distinguish between the noise footprint and the PLB-burst (comprised mainly within the range $20-50 \mathrm{kHz}$ ) that has been efficiently conserved after denoising, as 
Table 3: Denoising performance evaluation for signals channels 2 and 3 affected by a high noise level, with " $d b x$ " standing for the $x$-order of the Daubechies wavelet and " $D L$ " for the decomposition level.

\begin{tabular}{llllll} 
& & \multicolumn{2}{c}{ Channel 2} & \multicolumn{2}{c}{ Channel 3 } \\
\cline { 3 - 6 } & & $N_{\text {hits }}$ & $S N R\left(\cdot 10^{-4}\right)[d B]$ & $N_{\text {hits }}$ & $S N R\left(\cdot 10^{-4}\right)[d B]$ \\
\hline \multirow{3}{*}{$d b 8$} & $D L=6$ & 237 & 8.80 & 532 & 4.41 \\
& $D L=10$ & 30 & 8.16 & 250 & 3.89 \\
& $D L=20$ & 34 & -2.81 & 215 & -88.92 \\
\hline \multirow{3}{*}{$d b 10$} & $D L=10$ & 14 & 8.06 & 215 & 3.81 \\
& $D L=14$ & 13 & 7.99 & 202 & 3.73 \\
& $D L=20$ & 22 & -25.25 & 159 & -44.63 \\
\hline \multirow{2}{*}{$d b 20$} & $D L=10$ & 10 & 7.68 & 77 & 3.18 \\
& $D L=14$ & 10 & 7.60 & 63 & 3.10 \\
\hline \multirow{2}{*}{$d b 30$} & $D L=10$ & 6 & 8.07 & 26 & 2.95 \\
& $D L=14$ & 6 & 7.99 & 21 & 2.87 \\
\hline \multirow{2}{*}{$d b 45$} & $D L=7$ & 7 & 8.31 & 35 & 3.15 \\
& $D L=10$ & 5 & 8.02 & 11 & 2.84 \\
& $D L=14$ & 5 & 7.95 & 10 & 2.76 \\
\hline
\end{tabular}
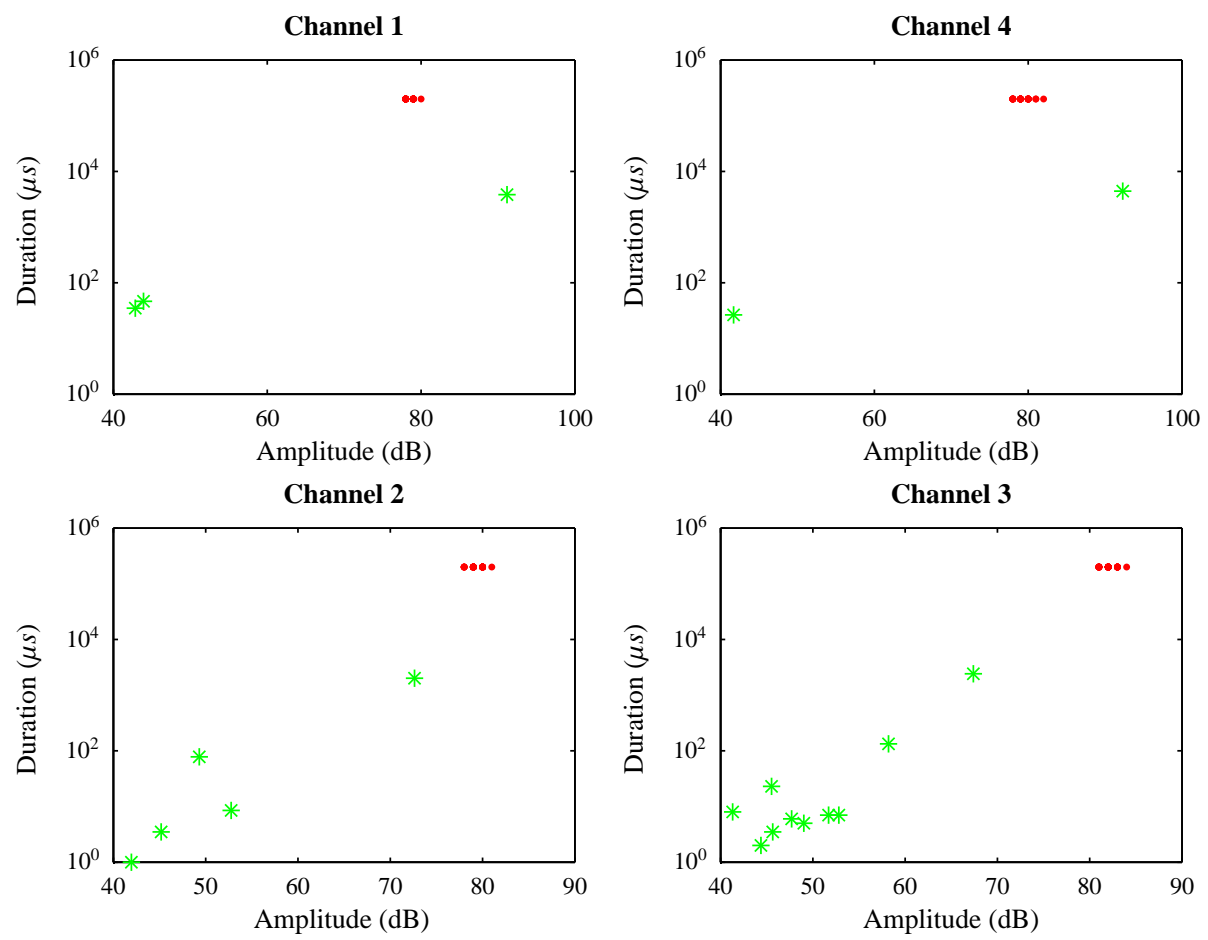

Figure 5: [Color representation] PLB bursts under a high noise level: Duration vs. Amplitude graphs of the detected hits by the AE system (•) and by the proposed approach $(*)$. The figures show that the saturation is eliminated.

we can observe in Figure 7(b). Accordingly, the frequency features (such as average frequency, centroid frequency ...) of a noisy signal are potentially altered, which may give erroneous information about the AE data and falsify further interpretations. The eventual alteration of the non-frequency AE-features is also studied. Table 4 shows some calculated AE features for raw and denoised signals of channels 1 and 4, as their raw PLB-bursts are available. It can 

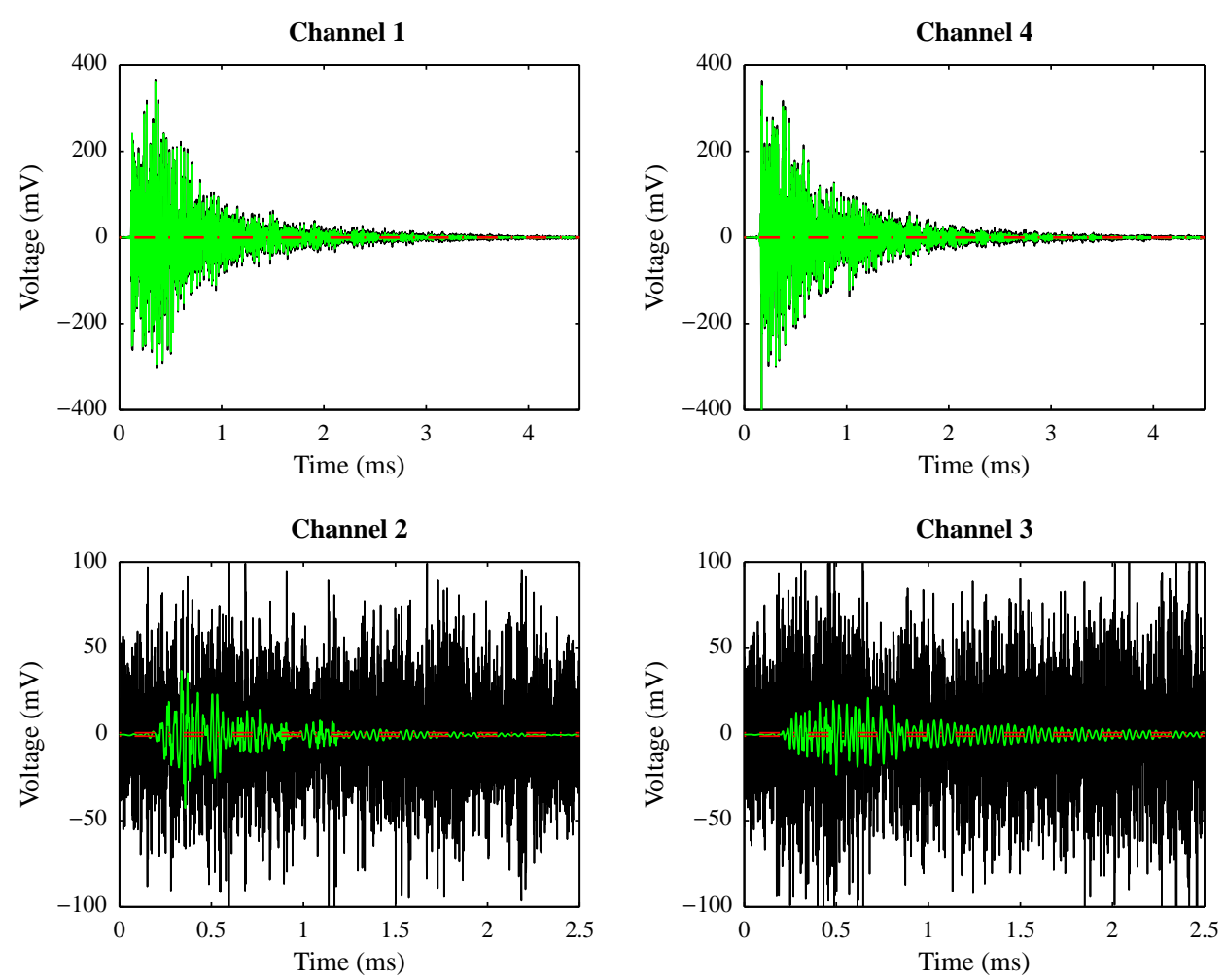

Figure 6: [Color representation] Comparison of the raw and denoised signals of the four channels after a PLB under a high noise level. Denoising using the "d $d b 45$ " wavelet and 14 decomposition levels. ( - Raw signals; $(-)$ denoised signals; and $\left(---_{-}\right)$threshold level.

be noted that the signal amplitudes are conserved and not altered by the denoising process. Obviously, the raw-signal durations are erroneous as they are equal to the predefined maximum duration ( $200 \mathrm{~ms})$, those of the denoised signals would be more correct. However, the number of counts of the PLB-bursts is very affected by denoising, as they are superimposed with the number of counts of the noise-signals. PAC-Energy is almost divided by two; the alteration of the duration and the number of counts is compensated by a conservation of the amplitude.

Table 4: Effect of denoising on non-frequency AE features.

\begin{tabular}{lllll}
\cline { 2 - 5 } & \multicolumn{2}{c}{ Channel 1 } & \multicolumn{2}{l}{ Channel 4 } \\
\cline { 2 - 5 } & Raw & Denoised & Raw & Denoised \\
\hline Amplitude $[\mathrm{dB}]$ & 91 & 91 & 92.5 & 92.3 \\
Duration $[\mu \mathrm{s}]$ & $2.10^{5}$ & 3832 & $2.10^{5}$ & 4421 \\
Counts & 28792 & 528 & 31333 & 605 \\
PAC-Energy $[\mu \mathrm{V} . \mathrm{s}]$ & 2350 & 1294 & 2400 & 1200 \\
\hline
\end{tabular}

\subsection{Complex AE signal: a high loading-rate tensile test under a high noise level}

A tensile test at a high loading-rate is performed on the same specimen. This test is performed after that of the PLB with a high noise level, so this latter is expected to be the same or higher. This configuration simulates an inservice-like loading under severe working conditions and results in complex experimental signals where continuous signals and AE transients could be superimposed. Practically, the test consists in applying an increasing tensile force 

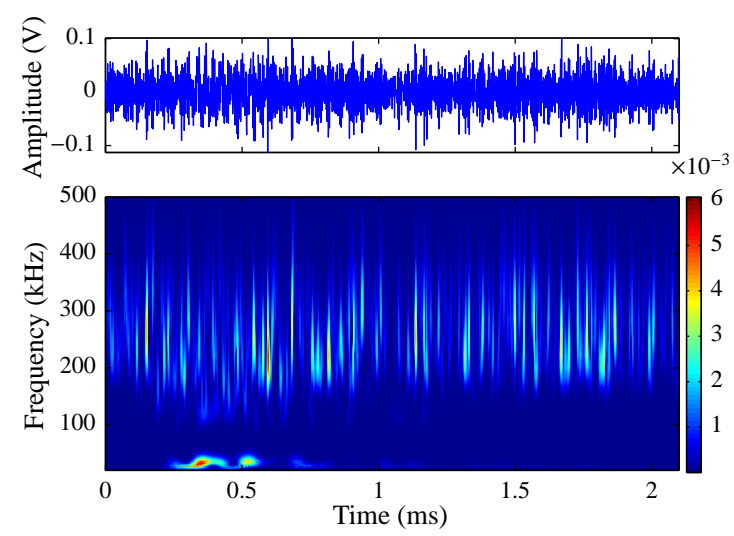

(a) Raw signal.
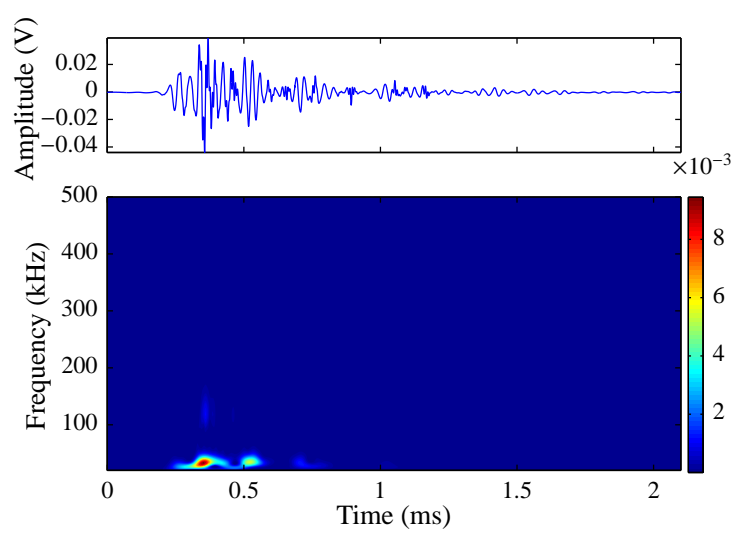

(b) Denoised signal.

Figure 7: [Color representation] Comparison of the WT diagrams of raw and denoised signals of channel 2 (PLB test under a high noise level).

(a ramp function) on the composite ring through the clamping jaws until the total failure. The applied force is going from $0 \mathrm{~N}$ to $60 \mathrm{kN}$ at a loading rate of $625 \mathrm{MPa} / \mathrm{s}$ [46].

\subsubsection{AE raw data}

Signals acquisition is launched before the beginning of the loading and is stopped after. The AE software records all the detected events produced in the material and determines all eventual hits. Features obtained by the AE system are presented hereafter and compared to those calculated after post-processing the signals by the proposed approach. Since channels 1 and 4 have quite similar responses (and similarly for channels 2 and 3), only the responses of channels 1 and 2 are considered hereinafter. A selection of features determined by the AE system is presented in Figure 8 .

Figures 8(a) and 8(b) show the amplitude of the detected hits and the applied force exerted by the machine on the specimen over time. First of all, as we can note, the amplitude increases with force, which begins to rise a little before the $4^{\text {th }}$ second; then it falls again when the force tumbles down shortly before the $7^{\text {th }}$ second with the complete failure of the ring. The hits detected outside the period of loading would correspond to noise as there was no other emission source in the material. We also remark that the level of their amplitudes is reduced after the rupture of the specimen. Indeed, on one hand, the noise generated by the hydraulic system is related to the applied force. When this latter falls, the fluid turbulence decreases, and therefore the noise level is reduced. On the other hand, the contact between the upper and lower clamping jaws is broken. So, channel 1 (analogously channel 4) is less impacted by noise after the failure of the composite ring. It should be also mentioned that the amplitude of the "noise hits" in channel 2 is higher than that in channel 1 since the level of noise is higher in channel 2, as shown also in the previous section. The next figures can prove this interpretation. Hits' durations obtained using the AE software are represented as a function of time (Figures 8(c) and 8(d)). For channel 1, all the hits recorded until the total failure of the specimen have durations equal to the maximum duration $(200 \mathrm{~ms})$. After the total failure of the specimen (end of loading), various hits are detected. Concerning channel 2, all the detected hits are equal to the maximum duration. This is a total saturation of the AE system throughout time. In the Duration-Amplitude graphs (Figures 8(e) and 8(f)), we note a saturation of hits at the maximum duration. As it was explained previously, the AE software has considered a number of signal segments with a duration of $200 \mathrm{~ms}$ as detected hits because the amplitude is stagnated above the threshold during a certain period within the test. These hits can be thus poorly separated. This potentially erroneous hit separation may be caused either by noise or by damage accumulation in the material leading to a high AE activity. This phenomenon is quite significant in channels 2 and 3 as they are more impacted by the generated noise. Figure 9 shows time signals of channels 1 and 2 as retrieved from the streaming file in order to be processed by the algorithm. 


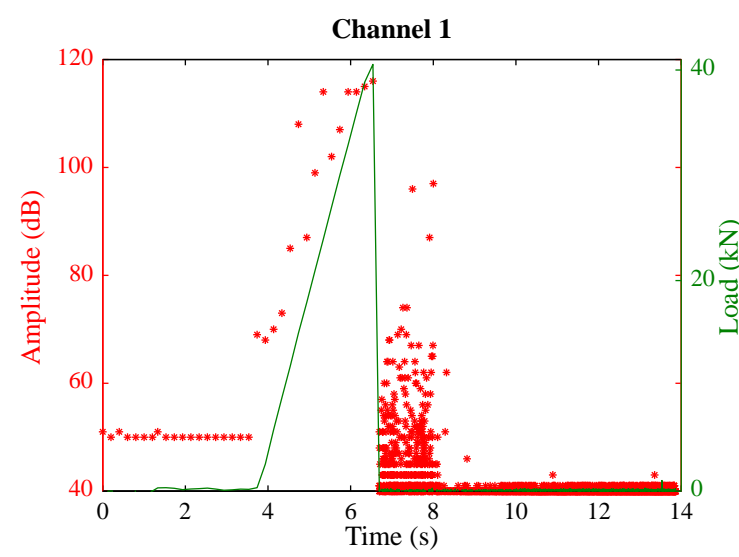

(a)

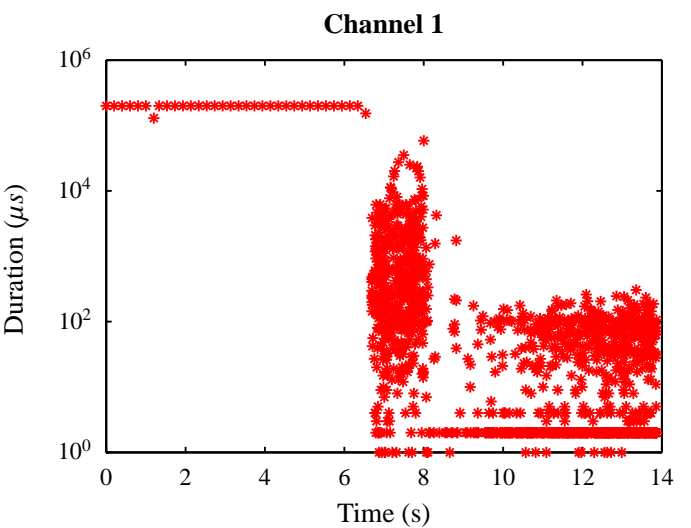

(c)

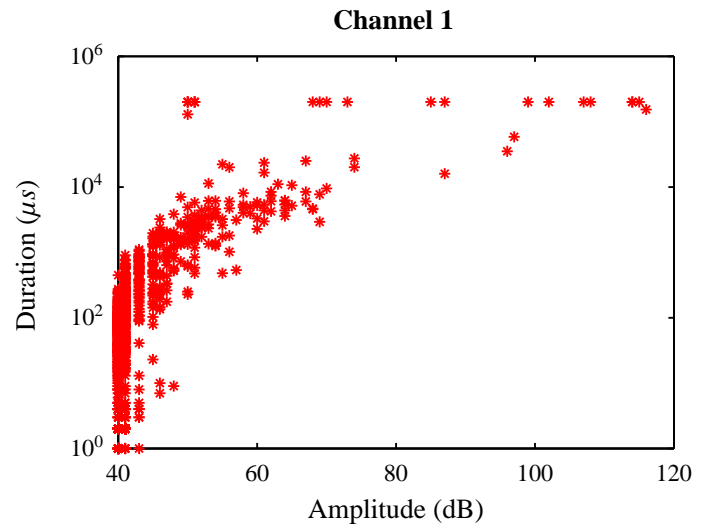

(e)

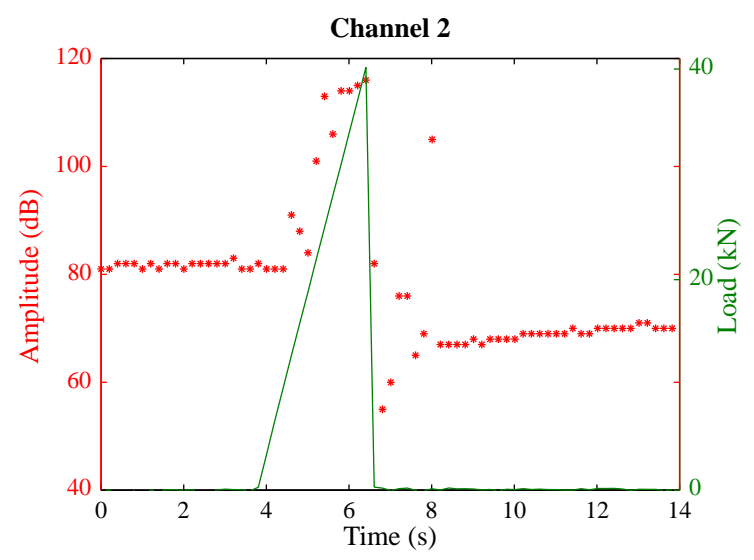

(b)

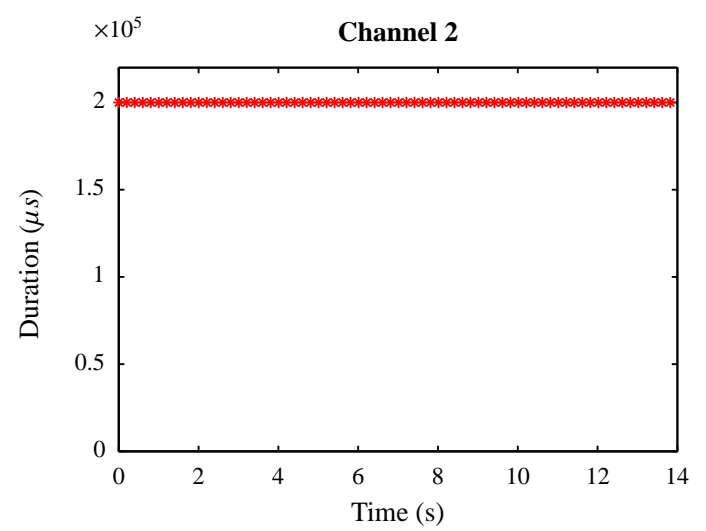

(d)

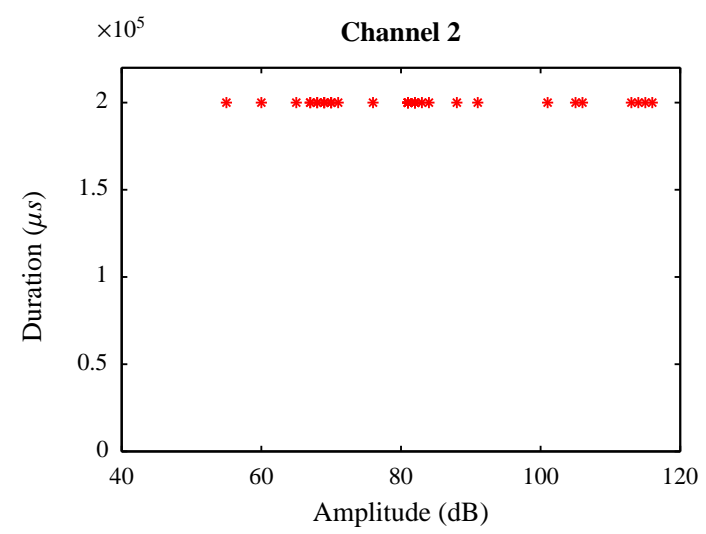

(f)

Figure 8: [Color representation] A selection of features retrieved from the AE data acquisition file of the tensile test for channels 1 and 2: (a) and (b) Amplitude and force over the acquisition time; (c) and (d) Duration over time; (e) and (f) Duration vs. Amplitude.

\subsubsection{Signal denoising}

The recorded time signals are post-processed and the resulting AE features are compared to those calculated by the method of the AE system. Initially, the signals of each channel are taken entirely; so that the denoising as well as the hit determination and separation are performed on each signal after loading it one shot. This strategy has the drawback 


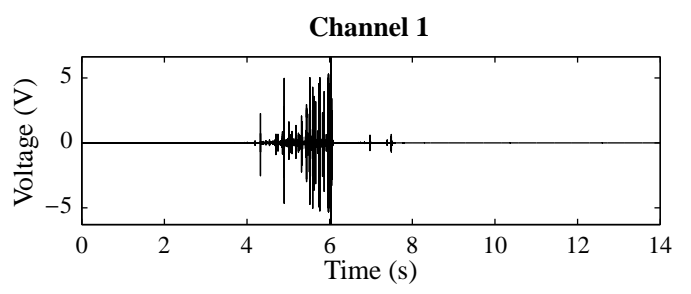

(a)

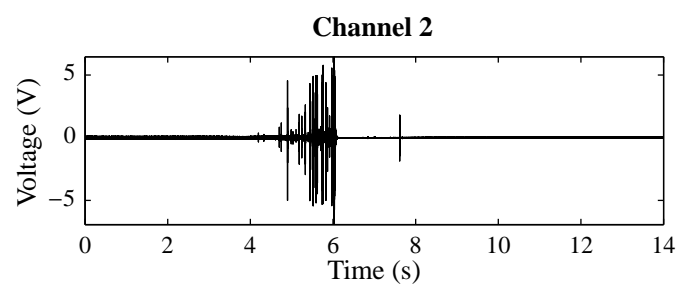

(b)

Figure 9: Raw time-signals obtained from channels 1 and 2 during the tensile test.

of requiring a lot of computer memory when treating massive data files as the size of each acquisition is large due to the sampling rate, which is generally from 2 to $5 \mathrm{MS} / \mathrm{s}$ in this kind of tests. Time signals of the four channels are denoised using different Daubechies wavelets, decomposition levels and threshold selection rules [33, 34], in order to find a suitable method efficiently applicable to this kind of tests. Table 5 summarizes the employed denoising parameters.

Table 5: Denoising parameters tested on the raw signals obtained for the tensile test under a high noise level.

\begin{tabular}{llll}
\hline Denoising parameters & Wavelet & Decomp. levels & Threshold selection rule \\
\hline DenPar1 & $d b 10$ & 7 & minimax (ch.1)/ heursure (ch.2) \\
DenPar2 & $d b 10$ & 7 & sqtwolog \\
DenPar3 & $d b 45$ & 7 & sqtwolog \\
DenPar4 & $d b 45$ & 14 & sqtwolog \\
\hline
\end{tabular}

Figure 10 shows a comparison of the Amplitude and Duration of both raw and denoised signals of channels 1 and 2. By comparing the different denoising methods, we remark that the hits obtained using 'DenPar1' are not well denoised; whereas 'DenPar3' is better than 'DenPar2' since it eliminates more efficiently noise hits detected before the loading. When increasing the decomposition level to 14 , no better improvement in signal denoising is noticed. Since increasing the decomposition level is time consuming, there is no need to adopt 7 levels. Accordingly, the best adjustment of the denoising process is obtained using the " $d b 45$ " wavelet, 7 decomposition levels, a soft thresholding with a selection rule of the universal threshold 'sqtwolog', and considering a non-white noise model.

Moreover, from Figures 10(a) and 10(c) (channel 1), we can note that the hits detected before and after the effective period of loading in the raw signal are now eliminated as they were representing the noise. As shown in Figures 10(b) and 10(d) (channel 2), although the noise hits are eliminated after the rupture of the ring, the amplitude of those detected before the start of the loading is reduced by about $10 \mathrm{~dB}$. Another important observation can be also taken from Figures 10(a) and 10(b): the amplitudes of the hits detected during the loading, which are associated to various AE evolving sources, are approximately conserved. So that the denoising process did not alter the effective AE information. Besides, in Figures 10(c) and 10(d) the hidden information by the effect of noise is now considerably revealed in channel 1 and especially in channel 2. However, some continuous emission still persists: about twelve hits during the loading have a duration equal to $200 \mathrm{~ms}$ (the Maximum Duration), which can be also observed in Figures 10(e) and 10(f) showing the Duration-Amplitude graphs.

\subsubsection{Signal segmentation}

In order to overcome the problem of loading heavy signal files and trying to totally eliminate the hits saturation, a signal segmentation is adopted. The signal to be processed is thus divided into short segments having equal durations of $0.5 \mathrm{~s}$. Then, each signal segment is successively denoised and potential hits are determined. This strategy has the advantage of surmounting the limitation of computer memory. The obtained results are presented in Figure 11 where the amplitude and duration of the hits detected after the signal segmentation are compared to those obtained previously by processing the entire signal using 'DenPar3'.

The most important ascertainment is that the saturation phenomena is now eliminated. All the separated hits have so a duration less than the pre-defined maximum duration. For channel 2, the hits detected before loading are greatly 


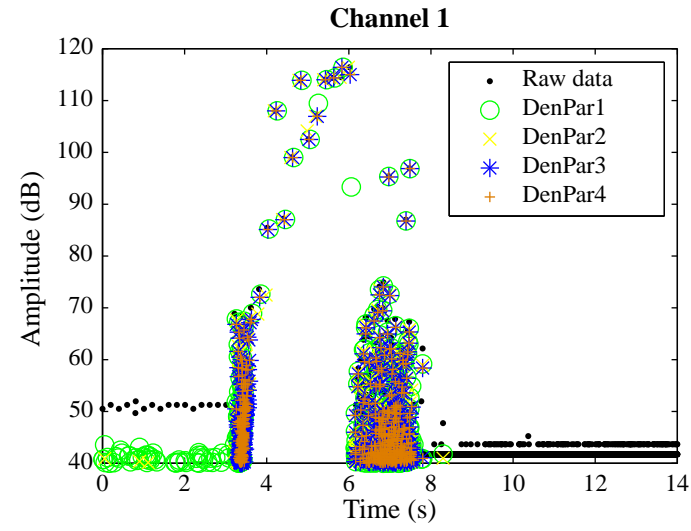

(a)

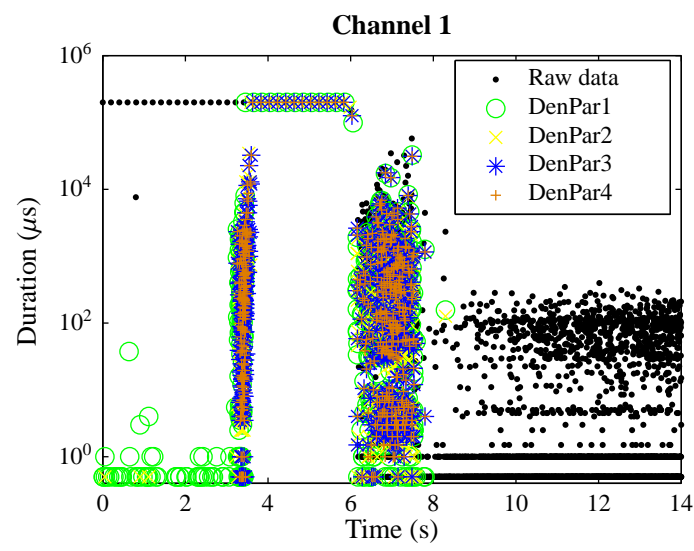

(c)

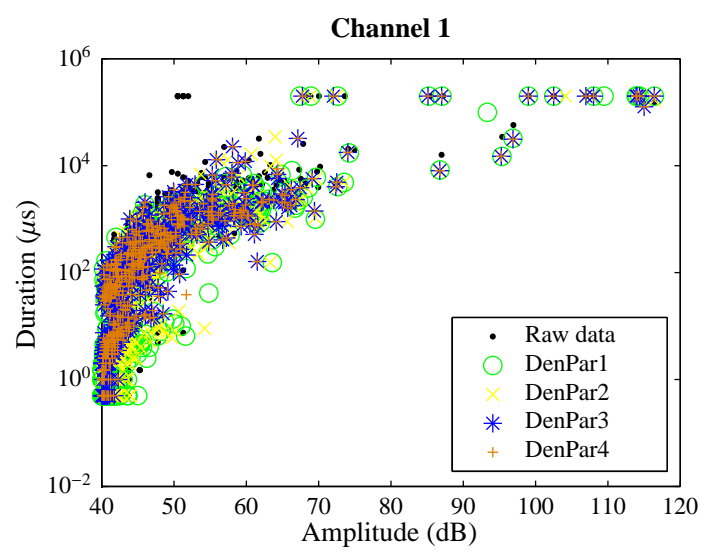

(e)

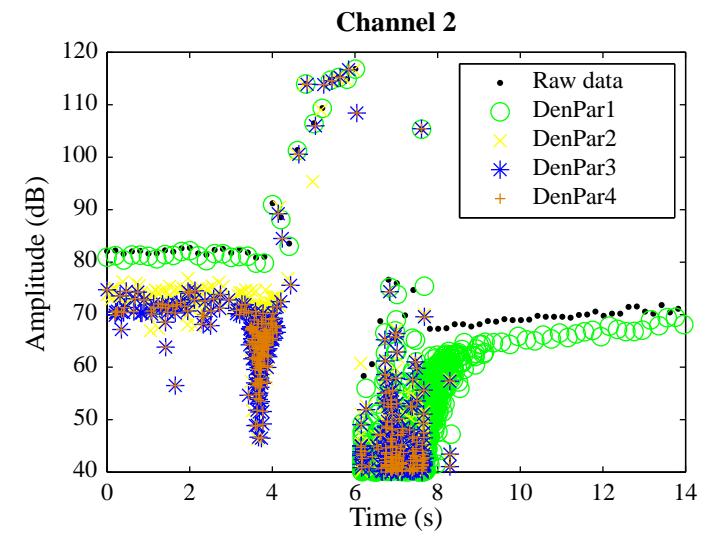

(b)

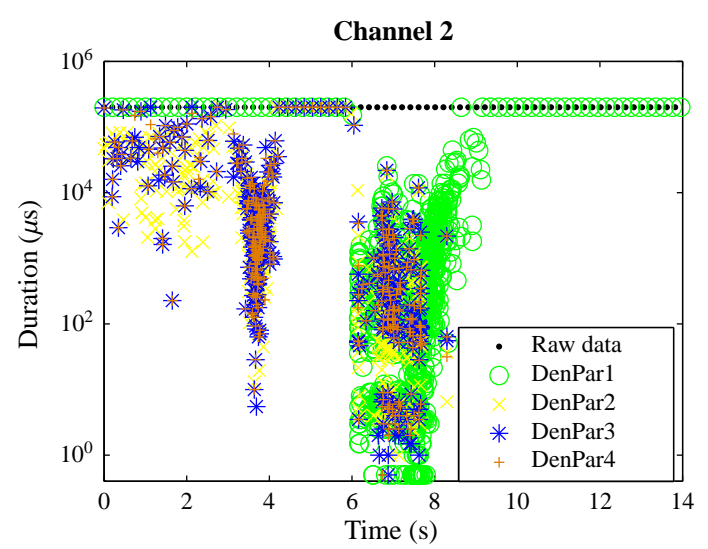

(d)

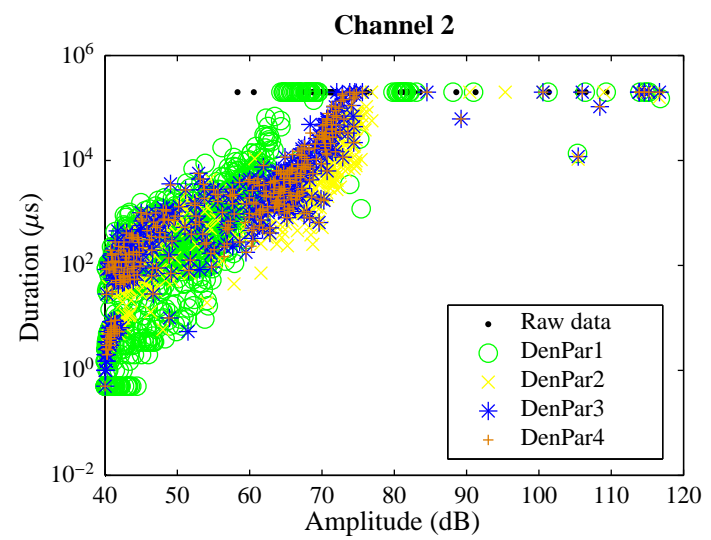

(f)

Figure 10: [Color representation] Comparison between the detected hits in the raw data (•) and those detected after denoising with different parameters. Signals processed entirely one-shot for channels 1 and 2. (a) and (b) Amplitude over the acquisition time; (c) and (d) Duration over the acquisition time; (e) and (f) Duration vs. Amplitude.

reduced. In fact, if the AE signal is processed entirely, the wavelet denoising procedure constructs an estimation of the noise model based on the full length of the signal, which could not be fairly accurate. Whereas, if the AE signal is segmented, the noise variation in each segment is less important than that in the entire signal. So, the noise 


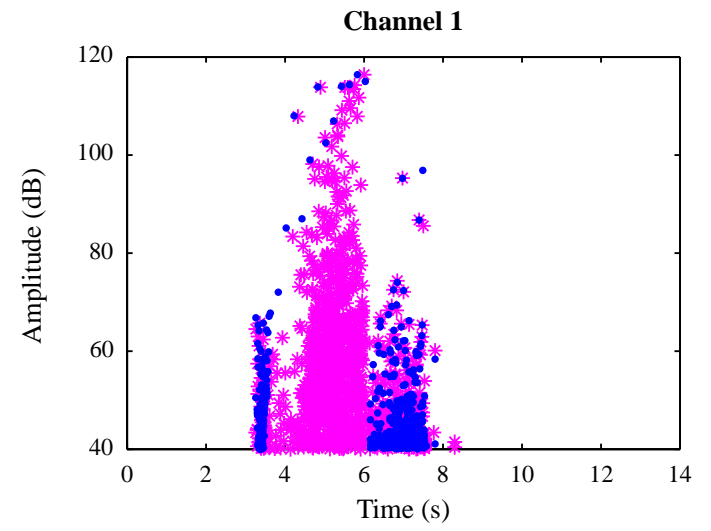

(a)

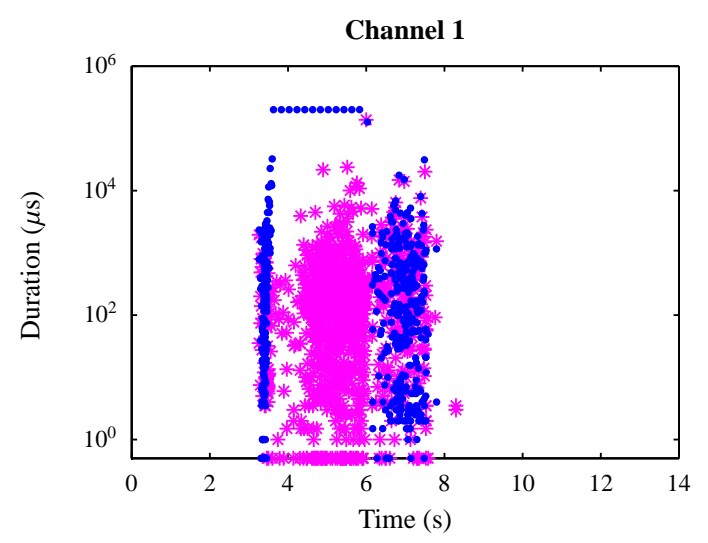

(c)

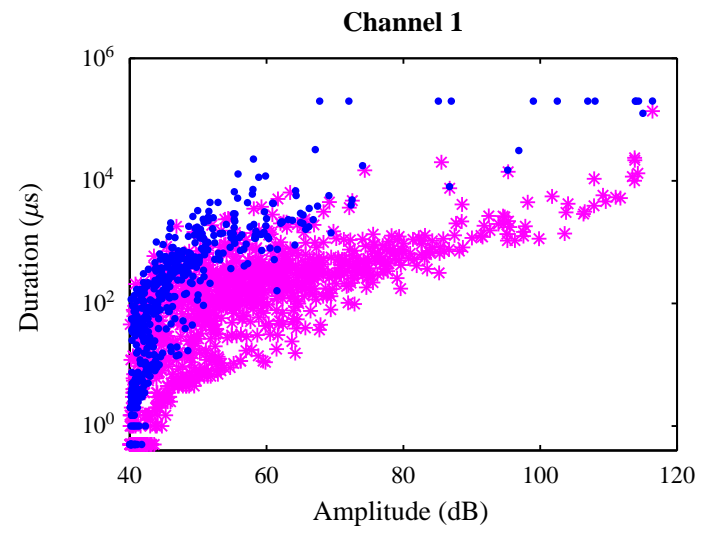

(e)

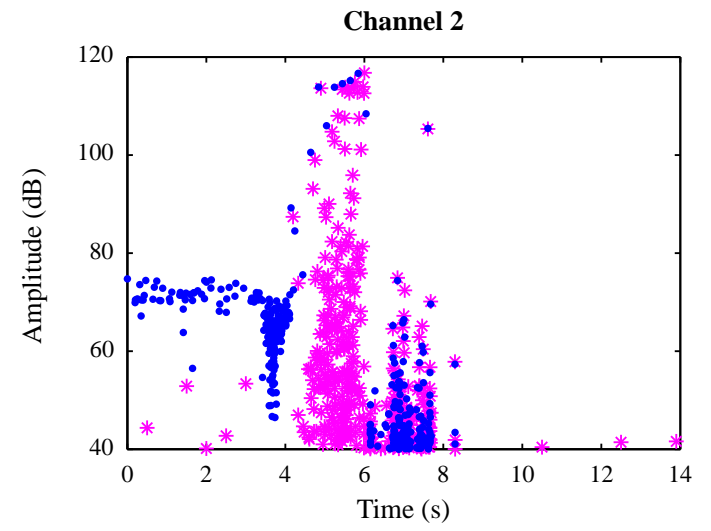

(b)

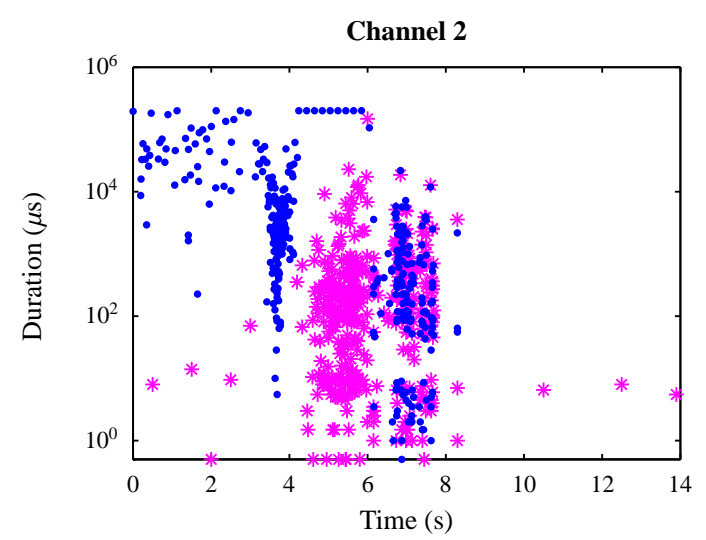

(d)

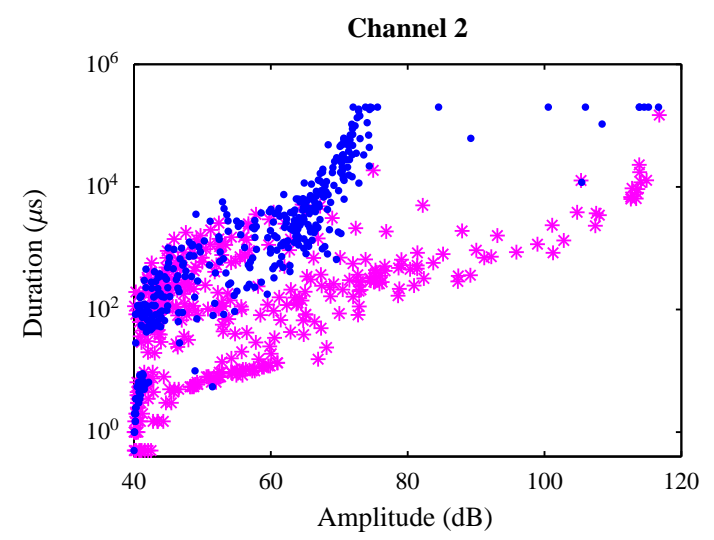

(f)

Figure 11: [Color representation] Comparison of the detected hits for channels 1 and 2 after a denoising using the parameters 'DenPar3': (•) Signals processed entirely one-shot; (*) signals segmented into 0.5 s-long segments. (a) and (b) Amplitude over the acquisition time; (c) and (d) Duration over the acquisition time; (e) and (f) Duration vs. Amplitude.

model is updated in each segment and is estimated more accurately, especially when dealing with non-stationary noise characteristics. It can be concluded that a blockwise wavelet denoising is more accurate to cope with highly non-stationary noise encountered in AE. An example of a signal-segment extracted from the response of channel 2 
during the loading is shown in Figure 12. The raw, non-segmented denoised, and segmented denoised signals are compared. It can be remarked that the signal obtained after segmentation is better denoised than that resulting after a one-shot denoising.

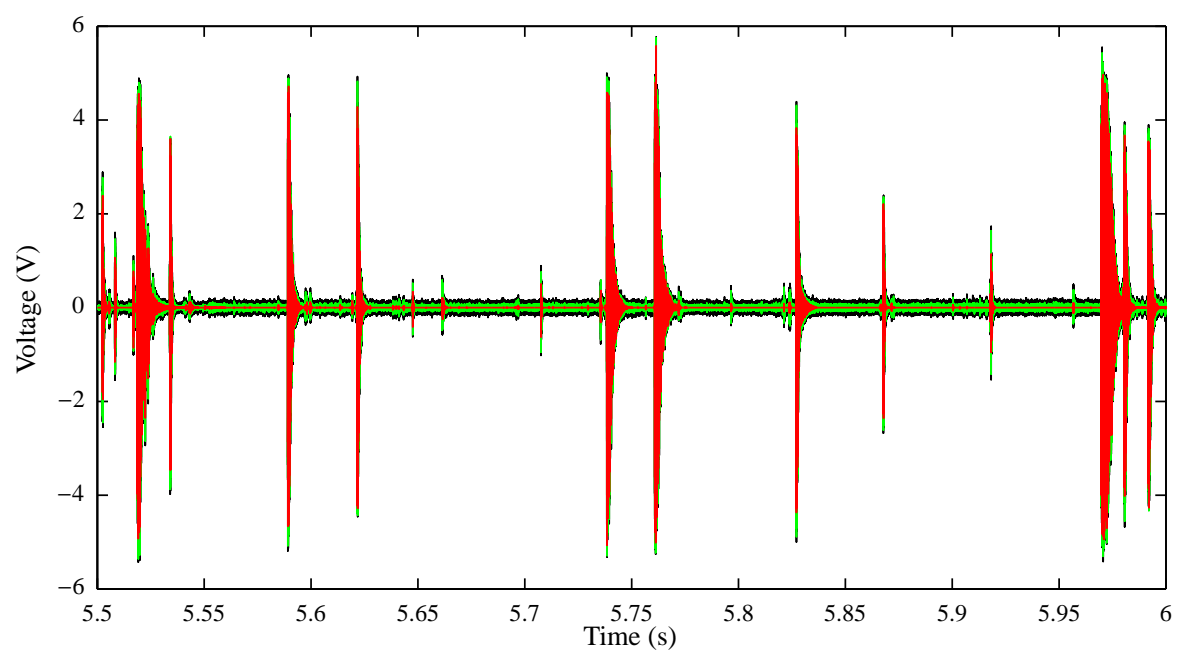

Figure 12: [Color representation] An example of a signal-segment extracted from channel 2: (—) Raw signal; ( $\longrightarrow$ ) non-segmented and denoised signal; and (—) segmented and denoised signal. Denoising using the parameters 'DenPar3'.

A deep observation of the hits' shapes that can be extracted by the algorithm leads to classify these hits into three categories whose samples are shown in Figure 13: a piece of hit, a typical burst of a natural AE source, and a relatively long hit exhibiting more than one waveform-packet that may be engendered by the superimposition of multiple AE hits. The separation of the first and the last hits seems to be not properly performed.

The hits belonging to the first category (Figure 13(a)) have a low number of counts and short durations; whereas the other two categories have medium and large number of counts, and medium and long durations. As shown in Figure 14, the truncated hits, which are located between 0 and 10 counts approximately appear as straight lines; that is to say multiple hits with equal counts are detected. This seems to be unlikely due to the complexity of the involved damage mechanisms, but may be engendered by a residual noise, wavelet artifacts, as well as a bad hits separation caused by inappropriate timing parameters (HDT, PDT and HLT). The proportions of the truncated hits are estimated at $40 \%$ for channel 1 and $49 \%$ for channel 2. The rest of the data would then contain the other two categories of hits (Figures 13(b) and 13(c)). The pattern recognition could be used here to regroup the truncated hits within a specific cluster. This issue is addressed in the next step of the proposed approach.

\subsubsection{Data clustering}

The AE data obtained after the above-mentioned denoising procedures using two processing parameterizations, namely one-shot with 'DenPar1' and segment-based with 'DenPar3', are analysed by the pattern recognition approach proposed in [39] and described in section 2.3. The clustering algorithm is used to determine the sequence of clusters in both cases. A number of clusters $\mathrm{K}=7$ is found to be the optimal value (based on the NMI criterion). Figure 15 presents the obtained sequences of clusters, where the vertical axis corresponds to the decimal logarithm of the cumulative occurrence of AE hits in a given cluster (called CSCA). These graphs allow locating the time of occurrence of each cluster, and following the temporal evolution of its activity. In Figure 15(a) obtained by using the one-shot denoising strategy, the four first clusters appear at the very beginning while the loading has not been applied yet (see the loading profile in Figures 8(a) and 8(b)). Furthermore, several clusters are highly activated later after the specimen failure. The damage scenario suggested by this sequence is thus unlikely. The sequence obtained in Figure 15(b) using the segment-based strategy is different. A first cluster starts at the same time as the AE acquisition (without loading) which is coherent with the activation of an AE source related to the 'structureborne' and 'fluidborne' noises. This cluster represents $62 \%$ of the total number of hits and may regroup those associated to external emission sources as well as 


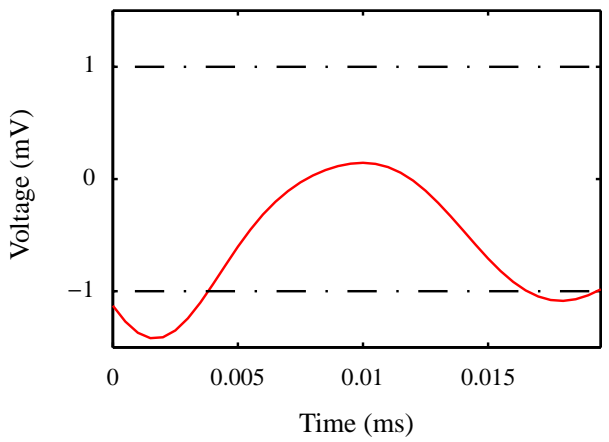

(a) Truncated hit.

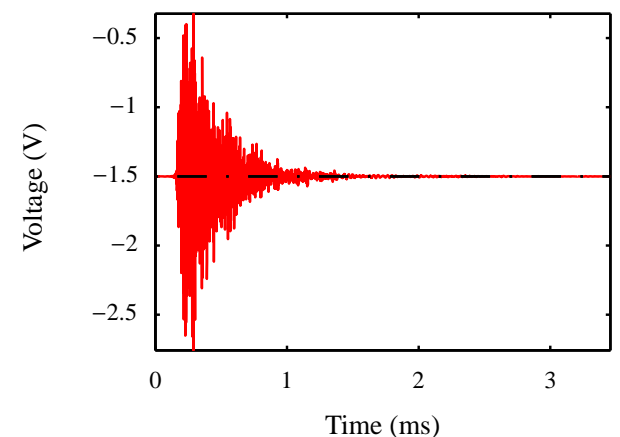

(b) Typical hit.

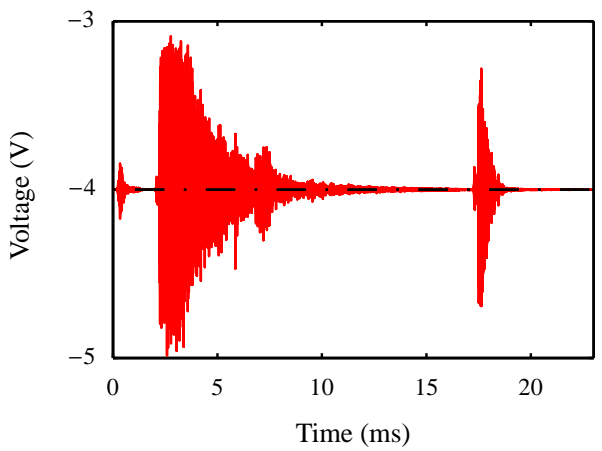

(c) Long hit.

Figure 13: Main three types of the extracted hits. (- - - ) threshold level.

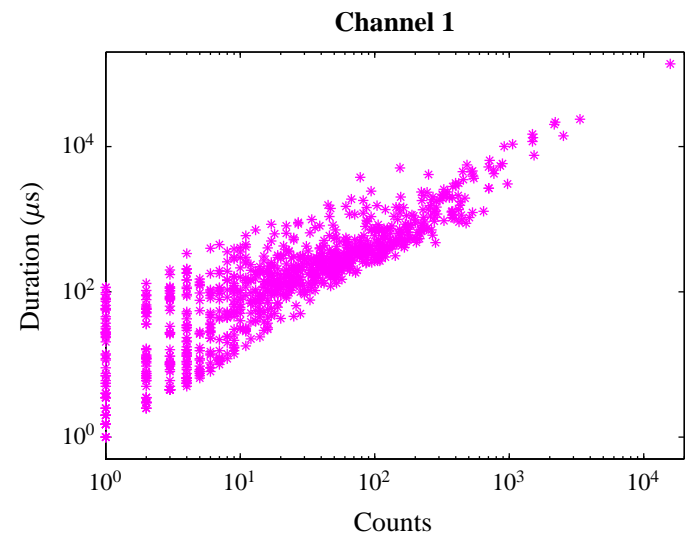

(a)

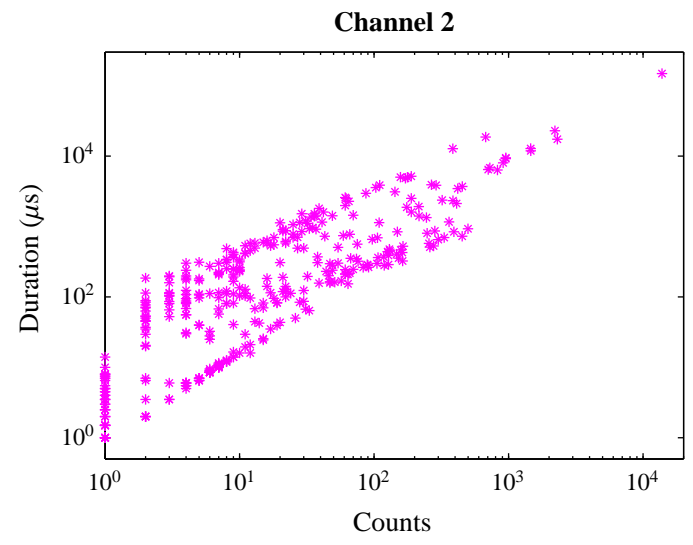

(b)

Figure 14: Duration vs. counts of the detected hits of channels 1 and 2 after the signal segmentation and denoising.

other meaningless hits. The first category of hits that was mentioned in the previous section, namely the truncated hits, is found to be included in this cluster after verification. Clusters 2, 3 and 4 start at the same time as the loading and are activated throughout the test. These clusters are thus associated to the activation of AE sources related for example to the friction between the specimen and the clamping-jaws. This friction is also observed after the specimen failure. 
The remaining clusters 5, 6 and 7 are probably associated to the material damage mechanisms as they appear during the specimen loading period. In CFRP composites, major damage mechanisms are matrix cracking, delamination, debonding, fiber cracking and fiber pull-out [9].

It can be concluded that the proposed algorithm leads to a better identification of the natural clusters in acoustic emissions and improves the interpretation of damage mechanisms.

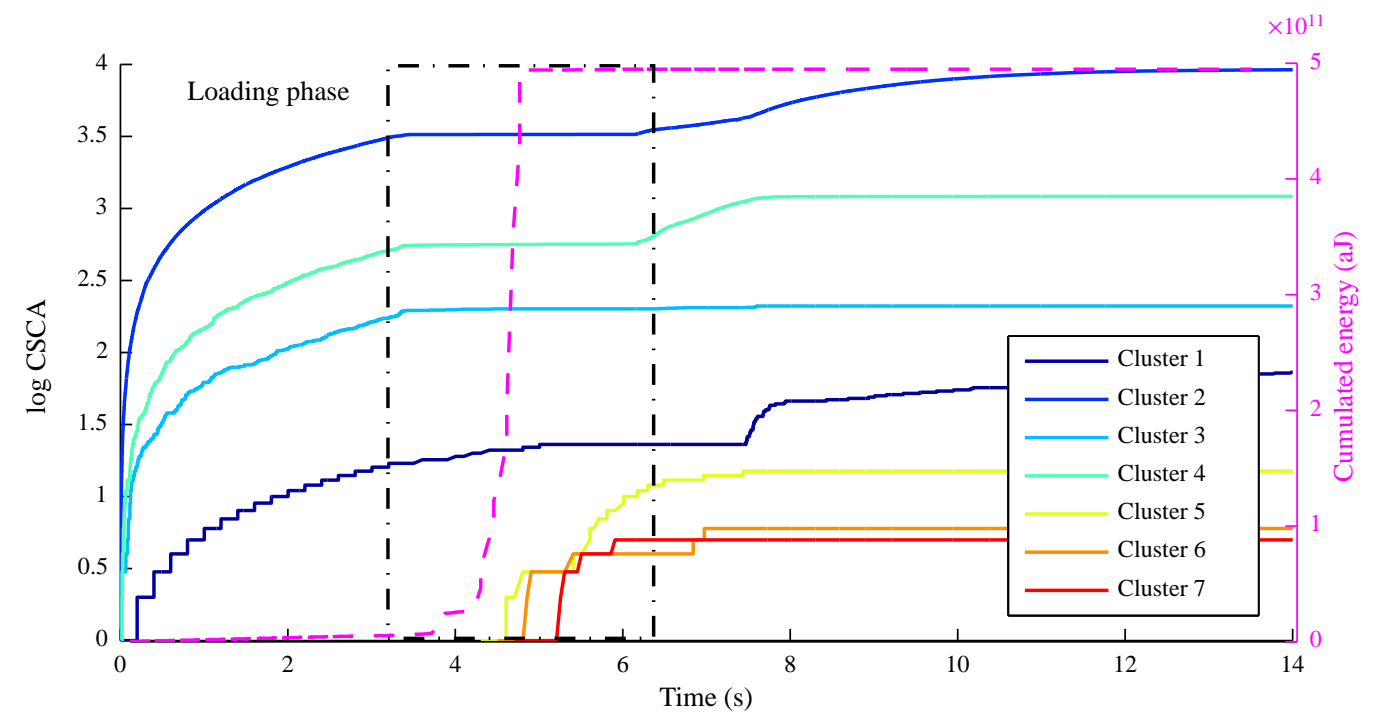

(a) Signals denoised entirely one-shot using the parameter 'DenPar1'. The sequence of damages is unlikely since some clusters start too early or activate later with respect to the loading phase (4-7s).

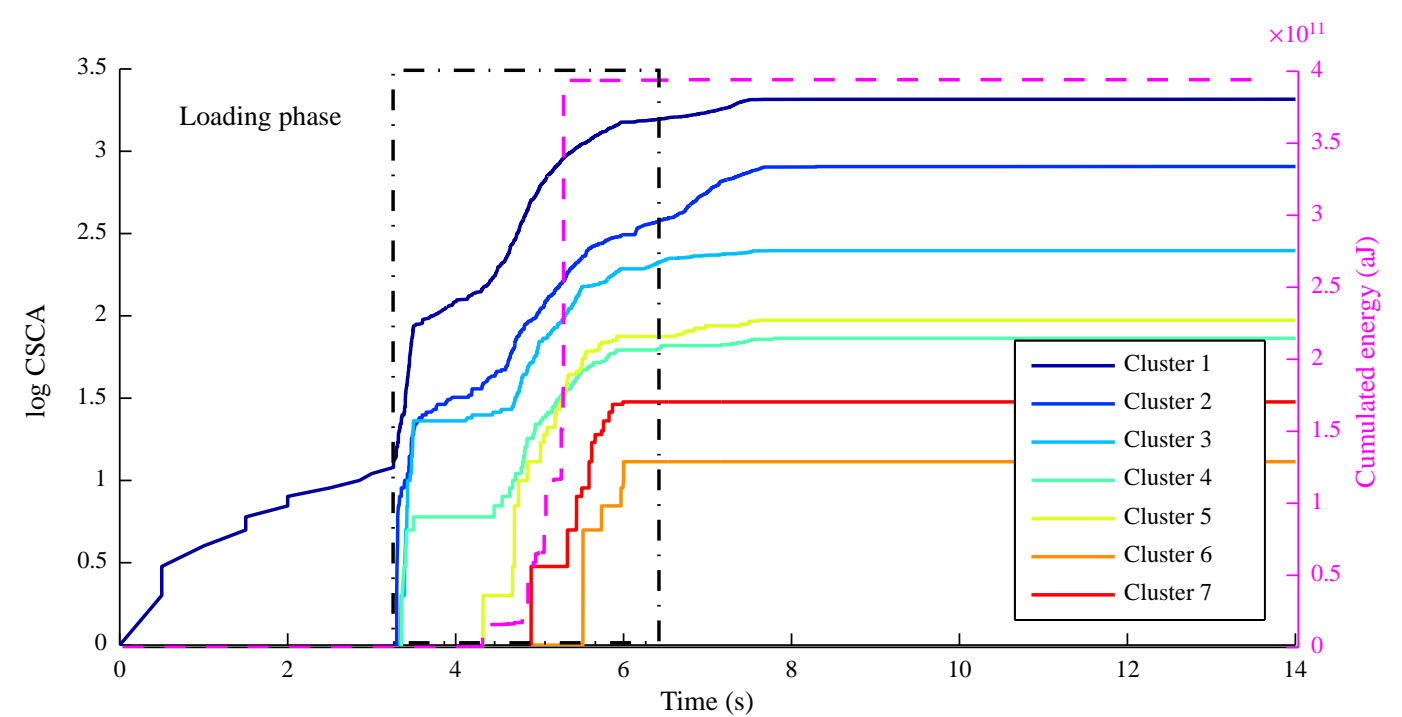

(b) Signals segmented and denoised using the parameter 'DenPar3'. The sequence of damages is enhanced as some clusters start and evolve during the loading phase.

Figure 15: [Color representation] Clustering sequences obtained using two processing parameterizations on the AE signals of all channels.

\section{Conclusion}

The problem of continuous AE in CFRP was addressed in this paper. Continuous signals produced by in-servicelike environment are post-processed. The proposed approach includes successive steps allowing the denoising of raw 
signals, hits determination, and AE features extraction. In order to assess its efficiency, the approach was tested on AE signals derived from experimental procedures. They firstly consist in a PLB applied on the surface of a composite ring under low and high noise levels. A complex AE signal is also created after a high loading-rate tensile test under a high noise level until the total failure of the specimen.

The proposed approach was able to improve the signals quality under different working conditions if the denoising parameters were properly set. It was also found that the extracted AE features that had been erroneous due to the noise, became coherent and exploitable. Moreover, it was also shown that the frequency features of a noisy signal are altered by the noise, which engenders erroneous information about the AE information. Using the proposed approach, the denoising process has eliminated considerably the noise contribution in the frequency content of the signal; whereas the frequency content of the PLB-burst was kept. The process of signal segmentation was able to substantially improve the hit determination and eliminate the hit saturation, especially when dealing with non-stationary noise in large signals recorded during high loading-rate tests. Besides, the signal segmentation was found to be of interest for pattern recognition. An appropriate hit detection algorithm leads to a better identification of natural clusters in AEs and improves the interpretation of damage mechanisms. In order to assess its reliability, the proposed approach can be applied on AE signals retrieved for example from fatigue tests with cycling loading performed on the studied composite ring. Such test case will be addressed in future works.

\section{Acknowledgment}

This work was supported by the Laboratory of Excellence ACTION through the program "Investments for the Future" managed by the National Agency for Research (references ANR-11-LABX-01-01) and by BPI France and the Franche-Comté Region through the project "Smart Composites".

\section{References}

[1] Q. Wu, F. Yu, Y. Okabe, K. Saito, S. Kobayashi, Acoustic emission detection and position identification of transverse cracks in carbon fiber reinforced plastic laminates by using a novel optical fiber ultrasonic sensing system, Structural Health Monitoring 14 (3) (2015) $205-213$.

[2] Y. Xu, R. Chen, Z. Liu, C. Shao, An acoustic-optical fiber NDE technique for interfacial debonding detection in FRP retrofitted structures, NDT \& E International 72 (0) (2015) 50 - 57

[3] I. Silversides, A. Maslouhi, G. LaPlante, Acoustic emission monitoring of interlaminar delamination onset in carbon fibre composites, Structural Health Monitoring 12 (2) (2013) 126-140.

[4] J. Berthelot, J. Rhazi, Acoustic emission in carbon fibre composites, Composites Science and Technology 37 (4) (1990) 411 - 428.

[5] R. Unnthorsson, T. Runarsson, M. Jonsson, Acoustic emission based fatigue failure criterion for CFRP, International Journal of Fatigue 30 (1) (2008) 11-20

[6] M. Sause, S. Horn, Simulation of acoustic emission in planar carbon fiber reinforced plastic specimens, Journal of Nondestructive Evaluation 29 (2) (2010) 123-142.

[7] I. Silversides, A. Maslouhi, G. LaPlante, Acoustic emission monitoring of interlaminar delamination onset in carbon fibre composites, Structural Health Monitoring (2013) 1475921712469994.

[8] C. Grosse, M. Ohtsu, Acoustic emission testing, Springer, 2008.

[9] D. Gay, Matériaux composites, Hermes, 2005.

[10] C. Scruby, An introduction to acoustic emission, Journal of Physics E: Scientific Instruments 20 (8) (1987) 946.

[11] T. Holroyd, The Acoustic Emission \& Ultrasonic Monitoring Handbook, Machine \& systems condition monitoring series, Coxmoor Publishing Company, Kingham, Oxford, UK., 2000.

[12] B. Harris, Fatigue and accumulation of damage in reinforced plastics, Composites 8 (4) (1977) 214-220.

[13] A. Mouritz, Non-destructive evaluation of damage accumulation, Fatigue in Composites, Woodhead Publishing Ltd., Cambridge (2003) $242-266$.

[14] B. Harris, Fatigue in composites: science and technology of the fatigue response of fibre-reinforced plastics, Woodhead Publishing, 2003.

[15] P. Hahn, T. Goss, A. Karlovsky, M. Scheerer, A. Pnninger, E. Ladsttter, An algorithm for life time prediction of fatigue loaded CFRPstructures derived from the rate of acoustic emission, in: Proceedings of the 4th European Workshop on Structural Health Monitoring, 2008, pp. 1173-1182.

[16] M. Lowe, G. Neau, M. Deschamps, Properties of guided waves in composite plates, and implications for NDE, in: Quantitative Nondestructive Evaluation, American Institute of Physics, 2004, pp. 214-221.

[17] E. Corporation, Noise Control in Hydraulic Systems (2002).

[18] J. Lin, L. Qu, Feature extraction based on morlet wavelet and its application for mechanical fault diagnosis, Journal of Sound and Vibration 234 (1) (2000) 135-148.

[19] S.-P. Song, P.-W. Que, Wavelet based noise suppression technique and its application to ultrasonic flaw detection, Ultrasonics 44 (2) (2006) 188-193.

[20] Y. Wang, Z. He, Y. Zi, Enhancement of signal denoising and multiple fault signatures detecting in rotating machinery using dual-tree complex wavelet transform, Mechanical Systems and Signal Processing 24 (1) (2010) 119-137. 
[21] M. R. Taha, A. Noureldin, J. Lucero, T. Baca, Wavelet transform for structural health monitoring: a compendium of uses and features, Structural Health Monitoring 5 (3) (2006) 267-295.

[22] D. Baleanu, H. Aydin, Advances in wavelet theory and their applications in engineering, physics and technology, InTech, 2012.

[23] A. Abbate, J. Koay, J. Frankel, S. Schroeder, P. Das, Application of wavelet transform signal processor to ultrasound, in: Ultrasonics Symposium, 1994. Proceedings., 1994 IEEE, Vol. 2, IEEE, 1994, pp. 1147-1152.

[24] Z. Hou, M. Noori, R. Amand, Wavelet-based approach for structural damage detection, Journal of Engineering Mechanics 126 (7) (2000) 677-683.

[25] H. Kim, H. Melhem, Damage detection of structures by wavelet analysis, Engineering Structures 26 (3) (2004) $347-362$.

[26] A. Marec, J.-H. Thomas, R. El Guerjouma, Damage characterization of polymer-based composite materials: Multivariable analysis and wavelet transform for clustering acoustic emission data, Mechanical Systems and Signal Processing 22 (6) (2008) $1441-1464$.

[27] B. Chen, Z. Zhang, C. Sun, B. Li, Y. Zi, Z. He, Fault feature extraction of gearbox by using overcomplete rational dilation discrete wavelet transform on signals measured from vibration sensors, Mechanical Systems and Signal Processing 33 (2012) $275-298$.

[28] M. Kharrat, M. N. Ichchou, O. Bareille, W. Zhou, Pipeline inspection using a torsional guided-waves inspection system. Part 1: Defect identification, International Journal of Applied Mechanics 06 (04) (2014) 1450034.

[29] C. Grosse, H. Reinhardt, M. Motz, B. Kroplin, Signal conditioning in acoustic emission analysis using wavelets, NDT.net 7 (9) (2002) 1-9.

[30] Y. Feng, S. Thanagasundram, F. Schlindwein, Discrete wavelet-based thresholding study on acoustic emission signals to detect bearing defect on a rotating machine, in: The Thirteen International Congress of Sound and Vibration. Vienna, Austria, 2006.

[31] X. Chiementin, D. Mba, S. Lignon, J. Dron, B. Charnley, Effect of the denoising on acoustic emission signals, Journal of Vibration and Acoustics 132 (3) (2010) 031009.

[32] A. Satour, S. Montrésor, M. Bentahar, R. Elguerjouma, F. Boubenider, Acoustic emission signal denoising to improve damage analysis in glass fibre-reinforced composites, Nondestructive Testing and Evaluation (2013) 1-15.

[33] D. L. Donoho, J. M. Johnstone, Ideal spatial adaptation by wavelet shrinkage, Biometrika 81 (3) (1994) $425-455$.

[34] D. L. Donoho, De-noising by soft-thresholding, IEEE Transactions on Information Theory 41 (3) (1995) 613-627.

[35] PCI-2 based AE system user's manual, Rev 3, Physical Acoustics Corporation (April 2007).

[36] V. Barat, Y. Borodin, A. Kuzmin, Intelligent AE signal filtering methods, Journal of Acoustic Emission 28 (2010) 109-119.

[37] V. Barat, D. Grishin, M. Rostovtsev, Detection of AE signals against background friction noise, Journal of acoustic emission 29 (2011) $133-141$.

[38] ASTM E1316-14, standard terminology for nondestructive examinations.

[39] E. Ramasso, V. Placet, M. Boubakar, Unsupervised consensus clustering of acoustic emission time-series for robust damage sequence estimation in composites, Accepted June 2015, IEEE Transactions on Instrumentation and Measurement.

[40] A. Strehl, J. Ghosh, Cluster ensembles — a knowledge reuse framework for combining multiple partitions, The Journal of Machine Learning Research 3 (2003) 583-617.

[41] E. Gustafson, W. Kessel, Fuzzy clustering with a fuzzy covariance matrix, in: IEEE Conf. on Decision and Control, 1978, pp. 761-766.

[42] N. Hsu, F. Breckenridge, Characterization and calibration of acoustic emission sensors, Materials Evaluation 39 (1) (1981) 60-68.

[43] M. Kharrat, E. Ramasso, V. Placet, M. L. Boubakar, A signal processing method for hits detection and separation in high ae activity systems: Application to composite materials under fatigue tests., in: EWSHM-7th European Workshop on Structural Health Monitoring, 2014, pp. 2298-2305.

[44] I. Daubechies, The wavelet transform, time-frequency localization and signal analysis, Information Theory, IEEE Transactions on 36 (5) (1990) 961-1005

[45] I. Daubechies, G. Teschke, Variational image restoration by means of wavelets: Simultaneous decomposition, deblurring, and denoising, Applied and Computational Harmonic Analysis 19 (1) (2005) 1-16.

[46] M. Kharrat, E. Ramasso, V. Placet, L. Boubakar, Acoustic emission in composite materials under fatigue tests: Effect of signal-denoising input parameters on the hits detection and data clustering, in: 31st Conference of the European Working Group on Acoustic Emission (EWGAE), At Dresde, Germany, 2014. 